\title{
ON FINITE SIMPLY REDUCIBLE GROUPS
}

\author{
L. S. KAZARIN AND V. V. YANISHEVSKII
}

Dedicated to the centenary of D. K. Faddeev's birth

\begin{abstract}
A finite group $G$ is said to be simply reducible ( $S R$-group) if it has the following two properties: 1) each element of $G$ is conjugate to its inverse; 2) the tensor product of every two irreducible representations is decomposed as a sum of irreducible representations of $G$ with multiplicities not exceeding 1 . It is proved that a finite $S R$-group is solvable if it has no composition factors isomorphic to the alternating groups $A_{5}$ or $A_{6}$.
\end{abstract}

\section{§1. INTRODUCTION}

Definition 1. A finite group $G$ is called an $S R$-group if it has the following two properties: 1) each element of $G$ is conjugate to its inverse; 2) the tensor product of every two irreducible representations is decomposed as a sum of irreducible representations of $G$ with multiplicities not exceeding 1 .

The class of $S R$-groups was introduced by E. Wigner $[\underline{6}$ and turned out to be fairly nontrivial. We note that $S R$-groups are of some interest in the theory of relation schemes (see [1, Chapter 2]). As pointed out in the book 4, there is no complete description of all $S R$-groups. We mention the important paper [5], in which some properties of $S R$ groups were investigated. Results on finite nonsupersolvable $S R$-groups were obtained in 3]. S. P. Strunkov (private communication) suggested that the $S R$-groups must be solvable. The main result of the present paper is a theorem giving a positive answer to the Strunkov conjecture under the additional assumption that the groups in question have no composition factors isomorphic to $A_{5}$ or to $A_{6}$.

In fact, we prove this statement for groups satisfying a weaker condition.

Definition 2. A finite group $G$ is called an $A S R$-group if it satisfies the following condition:

$\left.1^{\prime}\right)$ the tensor square of any irreducible representation of this group is decomposed as a sum of irreducible representations of $G$ with multiplicities not exceeding 1.

So, the definition of an $S R$-group is relaxed at two points: condition 1) (the groups satisfying this condition are said to be real) is lifted, and condition 2) is imposed only on the tensor squares of representations. Obviously, every $S R$-group is an $A S R$-group. The converse is false, because every Abelian group is an $A S R$-group whereas the Abelian $S R$-groups are elementary Abelian 2-groups. Examples of non-Abelian real $A S R$-groups are presented in $\S 8$.

The main result of the paper is as follows.

2000 Mathematics Subject Classification. Primary 53A04; Secondary 52A40. 52A10.

Key words and phrases. Group, subgroup, irreducible representation, character, tensor product, real element.

The first author was supported by RFBR (grant no. 05-01-01018). 
Theorem 1. Any finite ASR-group without composition factors isomorphic to $A_{5}$ or to $A_{6}$ is solvable.

It seems plausible that all finite $A S R$-groups are solvable. Theorem 1 is a consequence of the following more general statement.

Theorem 2. Let $G$ be a nonsolvable ASR-group of the smallest order. Then $G$ has a unique minimal normal subgroup $N$ that is the direct product of $m>1$ groups isomorphic to $A_{5}$ or to $A_{6}$. The subgroup $M$ of $G$ consisting of the elements that normalize every minimal normal subgroup of $N$ is normal in $G$, and the quotient group $G / M$ is a solvable $A S R$-subgroup of the symmetric group $S_{m}$ and acts transitively on the set of minimal normal subgroups of $N$.

In particular, any nonsolvable finite $A S R$-group, if it exists, must be of order divisible by 3600 .

One more result of this paper should be mentioned. Namely, Corollary 1, proved in $\S 2$, says that $k(G)^{3}<|G|$ for almost every simple non-Abelian group $G$. The only exceptions are provided by groups isomorphic to $L_{2}(q)$ for even $q$. Lemma 8 and Corollary 2 imply that every $A S R$-group is nonsimple.

The outline of the paper is as follows. In $\S 2$, we present some auxiliary results needed in the sequel. Also, that section contains information about the conjugacy classes of elements in simple non-Abelian groups. The proof of Corollary 1 (in which the greater part of the calculation is related to the most difficult case of the groups $L_{n}^{\varepsilon}(q), B_{n}(q)$, and $\left.C_{n}(q)\right)$ is used to present this information. In $\S 3$, we consider some general properties concerning the structure of $A S R$-groups and a minimal counterexample to Theorem 2 . In particular, we prove that a minimal counterexample to Theorem 2 has a unique minimal normal subgroup $N$ with solvable quotient group that is a direct product of isomorphic simple non-Abelian groups with certain restrictions on the degrees of irreducible representations. In $\S 4$, we use the results of [18] to exclude almost all simple groups that can arise as composition factors of $N$. The remaining set of groups consists of the families $L_{3}(q)$ and $U_{3}(q)$ for small values of $q$ and of the infinite family $L_{2}(q)$. In $\S 5$, we present necessary information on the correspondence between the irreducible characters of a group $G$ having a normal subgroup, the characters of this subgroup, and the characters induced from this subgroup (Clifford theory). Using this technique, we reduce the problem to the case where the composition factors of the group $N$ are isomorphic to one of the groups of the family $L_{2}(q)$. In $\S 6$, we discuss the characters of the group $P G L_{2}(q)$ and the action of its automorphism group on the set of characters of degree $q+1$. Finally, in $\S 7$, we prove Theorem 2, which implies Theorem 1. In $\S 8$, we give examples of groups illustrating the notion of an $A S R$-group and its relationship with the notion of an $S R$-group.

We adopt the standard notation, which is explained when required. All groups under consideration are finite. The terminology concerning group representations and characters follows [12. We consider only ordinary representations, i.e., representations over the field of complex numbers. In particular, an irreducible character is a character of an irreducible representation over the field of complex numbers. All necessary information concerning finite simple groups, their automorphism groups, and the corresponding notation can be found in [17.

For the reader's convenience, we recall some notation. We denote by $C_{n}$ a cyclic group of order $n$ and by $Q_{8}$ the quaternion group. The letter $p$ will denote a prime number. For a group $X$, we denote by $|X|_{p}$ the largest power of $p$ dividing the order of $X$ (the order of its Sylow $p$-subgroup).

For brevity, we use the unified notation for the groups $L_{n}(q)$ and $U_{n}(q)$ (respectively, for $S L_{n}(q)$ and $\left.S U_{n}(q)\right)$. Namely, we write $L_{n}^{\varepsilon}(q)=L_{n}(q)$ if the variable $\varepsilon$ (sign) takes 
the value + (that is, $\left.S L_{n}^{+}(q)=S L_{n}(q)\right)$ and $L_{n}^{\varepsilon}(q)=U_{n}(q)$ if $\varepsilon$ takes the value - (that is, $\left.S L_{n}^{-}(q)=S U_{n}(q)\right)$. In the case of groups of Lie type, the number $q$ is a power of a prime $p$.

Let $n$ be a positive integer. We denote by $p(n)$ the number of (unordered) partitions of $n$, i.e., the number of integer solutions of the equation $n=x_{1}+x_{2}+\cdots+x_{n}$, where $x_{1} \geq x_{2} \geq \cdots \geq x_{n} \geq 0$. The greatest common divisor of two integers $m$ and $n$ is denoted by $(m, n)$. By $\operatorname{Irr}(G)$ we denote the set of irreducible characters of $G$. If $G$ is a simple group of Lie type, then its Steinberg character (see [9]) is denoted by $\mathrm{St}_{G}$. The number of conjugacy classes of elements in $G$ is denoted by $k(G)$. The character of $G$ induced from a character $\zeta$ of a subgroup $H$ of $G$ is denoted by $\zeta^{G}$. The restriction of a character $\chi$ of $G$ to a subgroup $H$ of $G$ is denoted by $\left.\chi\right|_{H}$. The inner product of characters $\chi$ and $\Theta$ of $G$ is denoted by $[\chi, \Theta]$. We use an index at the inner product if we want to specify the group in which the inner product is calculated. For example, the Frobenius famous reciprocity law is stated as follows. For all characters $\chi$ of $G$ and $\Theta$ of a subgroup $H$ of $G$ we have:

$$
\left[\chi, \Theta^{G}\right]_{G}=\left[\left.\chi\right|_{H}, \Theta\right]_{H} .
$$

If the group in which the calculation is done is clear from the context, we drop the index at the inner product.

In Subsection 4.1 we shall need the formula for the dimension of irreducible representations of the groups $A_{n}$ and $S_{n}$ (the hook formula) corresponding to a given partition of $n$ and the related Young diagram. Relevant information can be found in the book 2]; see Theorem 20.1 therein.

\section{§2. Auxiliary Results}

The following results were proved in [18] (see Theorem 1, Lemma 2.1, and Theorem 2).

Lemma 1. Let $G$ be a finite non-Abelian simple group of Lie type over the field $F=$ $G F(q)$. Let $l$ be the untwisted rank of $G$. Then

$$
k(G) \leq(6 q)^{l} .
$$

If $k(G) \geq|G|_{p}$, where $p$ is the characteristic of $F$, then $G$ is isomorphic to $L_{2}(q)$ for an even $q$ with $k(G)=q+1$ or to $L_{2}(5)$ (in the latter case, $k(G)=5$ ).

Lemma 2. (i) Let $G$ be a solvable subgroup of $S_{n}(n \geq 3)$. Then $k(G) \leq \sqrt{3}^{(n-1)}$, and the order of $G$ does not exceed $24^{(n-1) / 3}$.

(ii) Let $G$ be a subgroup of $S_{n}$. Then $k(G) \leq 2^{n-1}$.

Proof. (i) The statement concerning the number of conjugacy classes of a solvable subgroup of $S_{n}$ is part of Theorem 2.2 in [16]. By Dixon's theorem [11], the largest order of a solvable subgroup of $S_{n}$ does not exceed $24^{(n-1) / 3}$.

(ii) This statement coincides with Theorem 2 in [18.

In some cases, we need sharper estimates for the groups $L_{n}^{\varepsilon}(q)$.

Lemma 3. Let $G$ be a finite non-Abelian simple group isomorphic to $L_{n}^{\varepsilon}(q)$, where $n \leq 6$. Then $k(G) \leq 2 q^{n-1}$, with the exception of $G=U_{4}(2)(k(G)=20)$ and $G=U_{5}(2)$ $(k(G)=47)$.

Proof. The number of conjugacy classes of $L_{n}^{\varepsilon}(q)$ was calculated for $n \leq 8$ in [19]. For the groups $L_{n}(q)$, the corresponding numbers are given explicitly as polynomials in $n$ and $q$ (see Table 4 in [19]). Studying the values of these expressions proves the lemma for the groups $L_{n}(q)$. 
For $U_{n}(q)$, we have a formula representing $k\left(U_{n}(q)\right)$ in terms of the values of the polynomials $\gamma_{n}^{\prime}=\gamma_{n}^{\prime}(q)$. Therefore, in this case, a preliminary calculation is necessary. The expressions for $k\left(U_{n}(q)\right)$ have the following form (formula (6.13) in [19]):

$$
k\left(U_{n}(q)\right)=1 /(q+1, n) \sum_{d_{1}, d_{2}} \phi\left(d_{1}\right) \phi_{2}\left(d_{2}\right) \gamma_{n /\left(d_{1} d_{2}\right)}^{\prime},
$$

where the summation is taken over all pairs of divisors $d_{1}$ and $d_{2}$ of $q+1$ for which the product $d_{1} d_{2}$ divides $n$. Here, $\phi$ is the Euler function, and $\phi_{2}(n)=n^{2} \prod\left(1-1 / p^{2}\right)$ (where $p$ runs through all prime divisors of $n$ ) is a generalization of the Euler function. We have $\phi_{2}(1)=\phi(1)=1$. The polynomials $\gamma_{n}^{\prime}$ are given explicitly (see Table 5 in [19]). Using these tables and the formula mentioned above, by way of an example we present here the calculation of $k\left(U_{3}(q)\right)$ and $k\left(U_{4}(q)\right)$.

We have the following values of $\gamma_{i}^{\prime}$ for $i \leq 4$ : $\gamma_{1}^{\prime}=1, \gamma_{2}^{\prime}=q+1, \gamma_{3}^{\prime}=q^{2}+q+2$, and $\gamma_{4}^{\prime}=q^{3}+q^{2}+3 q+2$. In the case where $(n, q+1)=1$, the summation is taken over a unique pair of divisors, $d_{1}=d_{2}=1$, and we see that $\gamma_{n}^{\prime}$ is the number of the conjugacy classes of the elements of $U_{n}(q)$, i.e., the above relation takes the form $k\left(U_{n}(q)\right)=\gamma_{n}^{\prime}$. Thus, it remains to consider the cases where $(n, q+1) \neq 1$.

Let $n=3$ and $(3, q+1)=3$. Then

$$
k\left(U_{3}(q)\right)=\frac{1}{3}\left(\gamma_{3}^{\prime}+\phi(1) \phi_{2}(3) \gamma_{1}^{\prime}+\phi(3) \gamma^{\prime}(1)\right)=\frac{1}{3}\left(q^{2}+q+12\right),
$$

which gives the value of $k\left(U_{3}(q)\right)$ for $(q+1,3)=3$.

Now, let $n=4$. Here we have more possibilities: $(4, q+1)=1,(4, q+1)=2$, and $(4, q+1)=4$. In the first case, we obtain the polynomial $\gamma_{4}^{\prime}$. If $(4, q+1)=4$, then we have the following pairs $\left\{d_{1}, d_{2}\right\}$ of divisors of $(4, q+1)=4$ over which the summation is taken: $\{1,1\},\{1,2\},\{2,1\},\{2,2\},\{1,4\}$, and $\{4,1\}$. Therefore, for $(4, q+1)=4$ we obtain

$$
\begin{aligned}
k\left(U_{4}(q)\right) & =\frac{1}{4}\left(\gamma_{4}^{\prime}+\left(\phi(2)+\phi_{2}(2)\right) \gamma_{2}^{\prime}+\left(\phi(4)+\phi_{2}(4)+\phi(2) \phi_{2}(2)\right) \gamma_{1}^{\prime}\right) \\
& =\frac{1}{4}\left(\left(q^{3}+q^{2}+3 q+2\right)+4(q+1)+17\right)=\frac{1}{4}\left(q^{3}+q^{2}+7 q+23\right) .
\end{aligned}
$$

If $(4, q+1)=2$, then the same calculation gives

$$
k\left(U_{4}(q)\right)=\frac{1}{2}\left(q^{3}+q^{2}+7 q+9\right) .
$$

Now, we present the remaining formulas for $n=5$ and $n=6$. Let $n=5=(q+1,5)$. Then

$$
k\left(U_{5}(q)\right)=\frac{1}{5}\left(q^{4}+q^{3}+3 q^{2}+4 q+31\right) .
$$

Finally, for $n=6$, we have

$$
\begin{array}{llll}
k\left(U_{6}(q)\right)=q^{5}+q^{4}+3 q^{3}+5 q^{2}+6 q+4 & \text { if } & (6, q+1)=1 ; \\
k\left(U_{6}(q)\right)=\frac{1}{2}\left(q^{5}+q^{4}+3 q^{3}+9 q^{2}+10 q+12\right) & \text { if } & (6, q+1)=2 ; \\
k\left(U_{6}(q)\right)=\frac{1}{3}\left(q^{5}+q^{4}+3 q^{3}+5 q^{2}+16 q+14\right) & \text { if } & (6, q+1)=3 ; \\
k\left(U_{6}(q)\right)=\frac{1}{6}\left(q^{5}+q^{4}+3 q^{3}+9 q^{2}+20 q+62\right) & \text { if } & (6, q+1)=6 .
\end{array}
$$

It is easy to prove that the conclusion of Lemma 3 is true for the groups $U_{n}(q)$, $3 \leq n \leq 6$. We show how to do this for $n=6$. It is easy to show that for $q \geq 2$ the expression $\gamma_{6}^{\prime}=q^{5}+q^{4}+3 q^{3}+5 q^{2}+6 q+4$ cannot be less than any of the remaining expressions for $k\left(U_{6}(q)\right)$. Therefore, it suffices to prove that $k\left(U_{5}\right) \leq 2 q^{5}$ for every integer 
$q \geq 2$. This statement is obvious for $q \geq 7$ because $\gamma_{6}^{\prime}$ is the $q$-adic expression of an integer less than $2 q^{5}$. Substituting $q=3,4,5,6$, we see that the inequality is valid. In the case where $q=2$, the inequality $\gamma_{6}^{\prime} \leq 2 \cdot 2^{5}=2^{6}$ is no longer true, but $2+1=3$, and therefore, we must prove that $\frac{1}{3}\left(2^{5}+2^{4}+3 \cdot 2^{3}+5 \cdot 2^{2}+16 \cdot 2+14\right) \leq 2^{6}$, which gives the result in the case in question. If $n=4$ or $n=5$, then the above possibility does not arise, which leads to the exceptions listed in the lemma.

Lemmas 1, 2, and 3 imply the following statement.

Corollary 1. Let $G$ be a non-Abelian simple group for which $|G|<k(G)^{3}$. Then $G$ is isomorphic to the simple group $L_{2}(q)$ for some even $q$.

Proof. Let $G$ be a simple group of Lie type with untwisted Lie rank $l$, and let $k(G)^{3} \geq|G|$. We use the estimate $k(G) \leq(6 q)^{l}$ proved in Lemma 1 .

1. If $G \simeq L_{n}^{\varepsilon}(q)$, then $l=n-1$, and it is easy to check that $|G| \geq q^{n(n-1)+(n-2) / 2}$ for $q \geq 3$, and $|G| \geq 2^{(n-1)^{2}} 3^{n-1}$ for $q=2$ and $n \geq 2$. Therefore,

$$
\begin{aligned}
& (6 q)^{3 n-3} \geq q^{n(n-1)+(n-2) / 2} \quad \text { for } \quad q \geq 3 \text { and } \\
& (12)^{3 n-3} \geq 2^{n(n-1)} \quad \text { for } \quad q=2 .
\end{aligned}
$$

If $q \geq 3$, then $6<q^{5 / 3}$, and so $n^{2}-8.5 n+7<0$, which gives $n \leq 7$. For $q \geq 4$, we have a sharper estimate, $6<q^{4 / 3}$, whence $n \leq 6$.

For $n=7$ and $q=3$, we can use Tables 4 and 5 in 19 to obtain the estimate $k(G) \leq 2 \cdot 3^{6}$ for the group $G=L_{10}^{\varepsilon}(q)$, because $(7,3-\varepsilon \cdot 1)=1$. Thus, we have $n \leq 7$ for $q \geq 3$. From the above and Lemma 3, we obtain $k(G) \leq 2 \cdot q^{n-1}$. Hence,

$$
\left(2 \cdot q^{n-1}\right)^{3} \geq q^{n(n-1)+(n-2) / 2} .
$$

It is easily seen that $n \leq 3$. Applying sharp estimates contained in [19] and in Lemma 3 , we prove the claim.

If $q=2$, then $n \leq 11$. A more thorough calculation of the order of the group shows that $n \leq 10$. For the groups $L_{n}^{\varepsilon}(q)$ with $n=10$, the number of conjugacy classes is given in Tables 2 and 5 in [19], because $(10, q-\varepsilon \cdot 1)=1$. It can be proved directly that, in both cases (for $\varepsilon=+$ and $\varepsilon=-$ ), $k(G)^{3}$ is considerably less than the order of the Sylow 2-subgroup of $G$.

The group $L_{n}(2)$ coincides with $G L_{n}(2)$, and the values of the conjugacy classes are given in Table 2 in [19] for $n$ as above. In all cases, we have $k\left(L_{n}(2)\right)<2^{n}$. Thus, it remains to consider the groups $U_{n}(2)$ for $n \leq 9$. If $n=9$, then we can use Table 5 in [19] (the computational algorithm is given in the proof of Lemma 3 ). If $n=8$ or $n=7$, then Table 5 gives exact values for $k\left(U_{n}(q)\right)$. If $n \leq 6$, then we can use Lemma 3. In each of the remaining cases we have $k(G)^{3}<|G|$.

2. If $G \simeq B_{n}(q)$ or $C_{n}(q)$, then $l=n$, and it is easy to check that $|G| \geq \frac{1}{2} q^{2 n^{2}+(n-2) / 2}$ for $q \geq 3$ and $|G| \geq 2^{2 n^{2}} 3^{n}$ for $q=2$ and $n \geq 2$.

Therefore,

$$
\begin{aligned}
& (6 q)^{3 n} \geq \frac{1}{2} q^{2 n^{2}+(n-2) / 2} \quad \text { for } \quad q \geq 3 \text { and } \\
& (12)^{3 n} \geq 2^{2 n^{2}} 3^{n} \quad \text { for } \quad q=2 .
\end{aligned}
$$

First, we consider the case where $q \geq 3$. Solving the above inequality for $q \geq 6$, we get $n \leq 3$. If $q \geq 4$, then $6 q \leq q^{7 / 3}$. Therefore, we have $n \leq 4$ for $q \in\{4,5\}$. Finally, for $q=3$, we obtain $18=6 q=2 \cdot 3^{2}$, which implies $n \leq 4$.

The cases where $q \geq 7$ and $n \geq 3$ are ruled out by direct calculation. Thus, the remaining values of $q$ belong to the set $\{3,4,5\}$, and $n \in\{2,3,4\}$. The case of $n=4$ can be ruled out by an easy supplementary calculation. Finally, the case where $n=3$ is 
possible only if $q=3$ and can be ruled out by [10, because $k\left(B_{3}(3)\right)$ and $k\left(C_{3}(3)\right)$ do not exceed $3^{4}$.

Applying the computer algebra system GAP with function NrConjugacyClasses(G), in which we use the function ProjectiveSymplecticGroup $(4, q)$ instead of G, we can easily rule out the remaining groups for $n=2$ (the groups $B_{2}(q)$ and $C_{2}(q)$ are isomorphic).

If $q=2$, then the inequality $k(G)^{3} \geq|G|$ implies $n<5$, and it remains to use [10].

3. Similarly, we can rule out the remaining groups of Lie type. For completeness, we note that $k\left({ }^{2} B_{2}(q)\right)=q+3, k\left({ }^{2} G_{2}(q)\right)=q+8, k\left({ }^{2} F_{4}(q)\right)=q^{2}+4 q+17$ for $q>2$, and $k\left({ }^{2} F_{4}(2)^{\prime}\right)=22($ see [18]).

4. Let $G$ be a sporadic simple group. By [10], the numbers of classes for such groups are as follows: $k\left(M_{11}\right)=10, k\left(M_{22}\right)=12, k\left(M_{23}\right)=17, k\left(M_{24}\right)=26, k(H S)=24$, $k\left(J_{1}\right)=15, k\left(J_{2}\right)=21, k\left(J_{3}\right)=21, k\left(J_{4}\right)=124, k\left(C o_{1}\right)=103, k\left(C o_{2}\right)=60, k\left(C o_{3}\right)=$ $42, k(M c L)=24, k(S u z)=47, k(H e)=38, k(H N)=54, k(T h)=86, k\left(F i_{22}\right)=69$, $k\left(F i_{23}\right)=103, k\left(F i_{24}^{\prime}\right)=120, k(B)=188, k(M)=194, k(R u)=38, k(L y)=53$. In this case, Corollary 1 can be verified directly.

5. If $G$ is isomorphic to the alternating group $A_{n}$, then, by Lemma 2 , we have $k(G) \leq 2^{n-1}$. Since $\left|A_{n}\right|=n ! / 2 \leq 2^{3(n-1)}$, we obtain $n ! \leq 2^{3 n-2}$. A direct calculation shows that $15 !>2^{40}$. On the other hand, taking logarithms, we see that $\log _{2} n ! \leq 3 n-2$. For $n \geq 16$, we obviously have $\log _{2} n ! \geq 4(n-15)+\log _{2} 15 !>4 n-60+40=4 n-20$. Therefore, $n \leq 17$. It is clear that $k\left(A_{n}\right) \leq 2 k\left(S_{n}\right)=2 p(n)$, where $p(n)$ is the number of partitions of $n$. A table of values of $p(n)$ for $n \leq 100$ is contained in [7, p. 53]. For $5 \leq n \leq 17$, the function $2 p(n)$ is majorized by the function $2^{n / 2+2}$. Consequently, it suffices to prove the inequality $n !=2\left|A_{n}\right| \geq 2^{3 n / 2+7}$ for $n \geq 6$. If $n \geq 11$, then it is easy to check that $n !>2^{3 n / 2+7}$. Therefore, we can restrict ourselves to the case where $n \leq 10$. The cases where $n=10$ and $n=9$ can be ruled out by direct calculation using the estimate $k\left(A_{n}\right) \leq 2 p(n)$. For $n=6,7$, or 8 , we need sharper values of $k\left(A_{n}\right)$.

Since $A_{n}$ contains only even permutations and every two permutations of the same cycle type are conjugate in $S_{n}$, we have one of the following two possibilities. Either the permutations that belong to $A_{n}$ and have the same cycle type are conjugate in $A_{n}$, or there exist two conjugacy classes of permutations in $A_{n}$ of a given cycle type. In either case, a (rough) estimate from above for the number of conjugacy classes in $A_{n}$ is given by twice the number of types of even permutations of an $n$-element set.

Let $n=8$. Then we have 12 cycle types of even permutations. We obtain $40320=$ $8 !>(2 \cdot 12)^{3}=13824$, whence $n=8$ is impossible.

Let $n=7$. Then there are 8 cycle types of even permutations. We have $5040=7$ ! > $(2 \cdot 8)^{3}=4096$, a contradiction.

Let $n=6$. We have $k\left(A_{6}\right)=7$ and $720=6 !>(7)^{3}=343$, and so this case is also ruled out.

If $n=5$, then $k\left(A_{5}\right)=5$ and $\left|A_{5}\right|<5^{3}$. Since $A_{5} \simeq L_{2}(4)$, we see that, in this case, our statement is valid.

The following useful result was obtained by Gallagher [13].

Lemma 4. Let $G$ be a group and $H$ a subgroup of $G$. Then

$$
k(H) /|G: H| \leq k(G) \leq|G: H| k(H) .
$$

If $H$ is a normal subgroup of $G$, then $k(G) \leq k(H) k(G / H)$.

Lemma 5. Let $G$ be a non-Abelian simple group. Then there exists an irreducible character $\chi$ of $G$ such that $\chi(1)^{3} \geq|G|$.

Proof. See [15]. 
Lemma 6. Let $L$ be a non-Abelian simple group of Lie type defined over a field $G F(q)$ of characteristic $p$. Then $L$ has an irreducible character $S t_{L}$, the degree of which is equal to the order of the Sylow p-subgroup of $L$.

Proof. See [9].

\section{§3. A minimal counterexample to Theorem 1}

The following two lemmas will be used repeatedly, and Lemma 8 plays a crucial role in the proofs of the main results of the present paper.

Lemma 7. (i) A quotient group of an ASR-group is an ASR-group.

(ii) The direct product of two ASR-groups is an ASR-group.

Proof. The first part follows from the fact that every representation of a quotient group can be regarded as a representation of the given group. Part (ii) follows from the fact that every irreducible representation of a direct product $G=A \times B$ is the tensor product of an irreducible representation of $A$ and an irreducible representation of $B$ (see [12, Theorem (4.21)]). In particular, every irreducible character of $G$ is a product $\varphi \psi$, where $\varphi \in \operatorname{Irr}(A)$ and $\psi \in \operatorname{Irr}(B)$. The latter remark will be used repeatedly in the sequel.

Lemma 8. Let $G$ be a non-Abelian ASR-group, and let $\chi$ be an irreducible character of $G$. Then $\chi(1)<k(G)$ and $|G|<k(G)^{3}$.

Proof. Since $G$ is an $A S R$-group, the tensor square of every irreducible representation has a decomposition as a sum of irreducible representations of $G$ with multiplicities at most 1. In particular, for an irreducible representation of $G$ with character $\chi_{0}$ of the largest degree $\chi_{0}(1)$, we obtain $\chi_{0}^{2}=\sum_{k} c_{k} \chi_{k}$, where the summation is taken over all irreducible characters of $G$, and $c_{k} \in\{0,1\}$. Hence,

$$
\chi_{0}(1)^{2}=\sum_{\chi_{k} \in \operatorname{Irr}(G)} c_{k} \chi_{k}(1) \leq k(G) \chi_{0}(1) .
$$

Since $G$ is non-Abelian, the inequality is strict. Thus, $\chi_{0}(1)<k(G)$ for an irreducible character $\chi_{0}$ of $G$ of the greatest degree. However, in this case, we have $\chi(1)<k(G)$ for every irreducible character $\chi$ of $G$.

By the Frobenius formula and the above, we have

$$
|G|=\sum_{\chi \in \operatorname{Irr}(G)} \chi(1)^{2}<k(G) \chi_{0}(1)^{2} \leq k(G)^{3} .
$$

The lemma is proved.

Corollary 2. Let $G$ be a nonsolvable ASR-group. Then for the degree of any irreducible representation of $G$ we have $\chi(1) \leq k(G)-2$.

Proof. Indeed, choosing a character $\chi_{0}$ of the largest degree and arguing as above, we obtain the inequality

$$
\chi_{0}(1)^{2} \leq \sum_{j=1}^{k(G)} \chi_{j}(1) \leq a \chi_{0}(1)+(k(G)-a-1)\left(\chi_{0}(1)-1\right)+1,
$$

where $a$ is the number of irreducible characters $\chi$ of $G$ of degree $\chi(1)=\chi_{0}(1)$. Since the number of different degrees of irreducible characters of a nonsolvable group is at least 3 (see [12, Corollary (12.6)]), we have $k(G)-a-1>0$. Hence, $\chi_{0}(1)\left(\chi_{0}(1)-k(G)+1\right) \leq$ $1-(k(G)-a-1)$. If $\chi_{0}(1)=k(G)-1$, then $G=G^{\prime}$, and therefore, there exists a quotient group $H$ of $G$ that is (by Lemma 7(i)) a simple non-Abelian $A S R$-group. By Corollary 1 , the group $H$ is isomorphic to $L_{2}(q)$ for some even $q$. However, the group 
$L_{2}(q)$ has characters of degrees $q+1, q, q-1$, and 1 (see [20]). This contradiction shows that $\chi_{0}(1)-(k(G)-1)<0$, which proves our statement.

From this point on, we assume that the $A S R$-group $G$ under consideration is a minimal counterexample to Theorem 1 . We prove some properties of $G$, which are used in the subsequent sections to prove that such a counterexample does not exist.

Lemma 9. Let $G$ be a nonsolvable $A S R$-group of the smallest order, and let $N$ be a minimal normal subgroup of $G$. Then $G / N$ is a solvable group, and $N$ is a socle of $G$, that is, the direct product $R_{1} \times R_{2} \times \cdots \times R_{m}$ of isomorphic non-Abelian simple groups. The group $G$ is a subgroup of the wreath product $\mathfrak{G}=A<S$, where $A=\operatorname{Aut}(L)$ for a simple non-Abelian group $L$ isomorphic to $R_{1}$, and $S$ is a solvable subgroup of the symmetric group of degree $m$, acting transitively on the set of minimal normal subgroups of $N$.

Proof. Lemma 7 (i) implies that, for every normal subgroup $N$ of $G$, the quotient group $G / N$ is also an $A S R$-group. Since the group $G$ is minimal, the group $G / N$ is solvable if $N \neq 1$. We assume that $G$ has two distinct minimal normal subgroups, say $M$ and $N$. Since $G / M$ and $G / N$ are solvable by the above, $G$ is also solvable, because

$$
G \simeq G /(M \cap N) \leq G / M \times G / N
$$

Thus, $G$ has a unique solvable minimal subgroup $N$, i.e., $N$ is the socle of $G$. Corollary 1 shows that $N$ is a proper nonsolvable subgroup of $G$.

Since $C_{G}(N)$ is normal in $G$ and $C_{G}(N) \cap N=Z(N)$ is a solvable normal subgroup of $G$, we see that $C_{G}(N) \cap N=1$. From the above, it follows that $C_{G}(N)=1$, so that $G \subseteq \operatorname{Aut}(N)$.

Since, obviously, $N$ is a characteristically simple group, we have

$$
N=R_{1} \times R_{2} \times \cdots \times R_{m},
$$

where the $R_{i}$ are isomorphic non-Abelian simple groups $(i \in\{1,2, \ldots, m\}, m \geq 1)$ and the quotient group $G / N$ is a solvable $A S R$-group.

The structure of the automorphism group of a characteristically simple non-Abelian group is well known. This automorphism group is a subgroup of the wreath product $\mathfrak{G}=\operatorname{Aut}(L)$ \ $S$, where $L \simeq R_{1}$ is a non-Abelian simple group and $S$ is a subgroup of the symmetric group $S_{m}$, acting transitively on the set of minimal normal subgroups of $N$. Since $G / N$ is solvable, we conclude that $S$ is a solvable subgroup of $S_{m}$. The lemma is proved.

We preserve the notation of Lemma 9 until the end of the proof of Theorems 1 and 2 .

Lemma 10. Let $a=|\operatorname{Out}(L)|$, and let $\chi_{0}$ be an irreducible character of $L$ having the greatest degree. Then

$$
\chi_{0}(1) \leq \sqrt{3} a k(L) \leq 1.733 \cdot a k(L) .
$$

Proof. It is easily seen that $G$ has a normal subgroup $M$ (the intersection of the base of the wreath product $\mathfrak{G}=A$ \& $S$ with $G$ ) that normalizes each subgroup $R_{i}, i \in$ $\{1,2, \ldots, m\}$, and satisfies the conditions $|M: N| \leq a^{m}$ and $G / M \simeq S$.

By Lemma 4, we have

$$
k(M) \leq|M: N| k(N)=a^{m} k(L)^{m} .
$$

Using Lemma 2(i) and Lemma 4, from (1) we deduce that

$$
k(G) \leq(2 a k(L))^{m} .
$$


Since $\chi_{0}$ is an irreducible character of the group $L \simeq R_{1}$ of the largest degree, we see that $\Theta_{0}=\chi_{0}^{m}$ is an irreducible character of $N=R_{1} \times R_{2} \times \cdots \times R_{m}$. By the Frobenius reciprocity theorem, we obtain

$$
\left[\left.\Theta_{0}^{G}\right|_{N}, \Theta_{0}\right]_{N}=\left[\Theta_{0}^{G}, \Theta_{0}^{G}\right]_{G}>0 .
$$

Therefore, there exists an irreducible character $\Phi_{0}$ of $G$ such that the restriction $\left.\Phi_{0}\right|_{N}$ contains $\Theta_{0}$. In particular,

$$
\Phi_{0}(1) \geq \chi_{0}(1)^{m} .
$$

Relations (2) and (3) and Lemma 8 yield

$$
\chi_{0}(1)^{m} \leq \Phi_{0}(1) \leq k(G) \leq(2 a k(L))^{m} .
$$

Thus,

$$
\chi_{0}(1) \leq 2 a k(L),
$$

where $\chi_{0}$ is an irreducible greatest degree character of $L$ and $a=|\operatorname{Out}(L)|$.

By Lemma 2(i), we have $k(G / M) \leq 3^{(m-1) / 2}$. Therefore, we can replace the constant $2^{m}$ by $3^{m / 2}$ in inequality (2). The resulting inequality takes the form

$$
\chi_{0}(1) \leq \sqrt{3} a k(L) \leq 1.733 \cdot a k(L),
$$

which proves the lemma.

\section{$\S 4$. REDUCTION}

In this section, we reduce the general situation to the case where the simple normal subgroup $R_{1} \simeq L$ of the minimal normal subgroup $N$ of $G$ is a classical simple group of small dimension.

By the classification theorem, a non-Abelian simple group is either a simple group of Lie type, or an alternating group, or one of the 26 sporadic simple groups. We use Lemma 10 and relation (4) obtained in the preceding section and consider each of these possibilities for $L$ separately.

4.1. Alternating groups. For $n \geq 7$, inequality (4) takes the following form:

$$
\chi_{0}(1) \leq 4 p(n),
$$

where $p(n)$ is the number of partitions of $n$ and $\chi_{0}$ is an irreducible character of $A_{n}$ of largest degree. Let $L=A_{n}$ be an alternating group satisfying (4). Then $n \leq 6$.

Lemma 11. Let $L=A_{n}$ be an alternating group satisfying inequality (4). Then $n \leq 6$.

Proof. First, we find all alternating groups satisfying (4). By Lemma 6 , the group $L$ has a character with degree greater than $|L|^{1 / 3}=(n ! / 2)^{1 / 3}$. Therefore, we obtain the inequality $n ! \leq 128 p(n)^{3}$. Since $4 p(n)<2^{n}$ for $n \geq 5$, we obtain $n !<2^{3 n+1}$, which implies $n \leq 21$. Using the fact that, for $n>7$, the group $L=A_{n}$ has an irreducible character $\varphi$ of degree $n(n-1)(n-3)(n-6) / 8$ corresponding to the partition $n=(n-4)+3+1$ (see [2, Theorem 20.1]), we see that $\varphi(1)>4 p(n)$ for $9 \leq n \leq 21$. Therefore, $n \leq 8$.

The group $L=A_{8}$ is isomorphic to $L_{4}(2)$ and has 14 irreducible characters. We have $\chi_{0}(1)=70$ and $a=2$. Therefore, $\chi_{0}(1)>4 \cdot 14=2 a k(L)$, and we may assume that $n=7$.

The group $L=A_{7}$ has 9 irreducible characters and $\chi_{0}(1)=35$. Applying Lemma 10, we obtain $35=\chi_{0}(1) \leq 1.733 \cdot 2 \cdot 9<34.2$, a contradiction. The lemma is proved.

Remark. The group $A_{5}$ is isomorphic to $L_{2}(4)$, and $A_{6}$ is isomorphic to $L_{2}(9)$. Therefore, all possible exceptions are classical groups. 


\subsection{Sporadic simple groups.}

Lemma 12. The group $L$ is not a sporadic simple group.

Proof. Using the Frobenius theorem, we represent inequality (4) in the form

$$
|L|=\sum_{\chi \in \operatorname{Irr}(L)} \chi(1)^{2} \leq k(L) \chi_{0}(1)^{2} \leq 4 a^{2} k(L)^{3} .
$$

We recall that for sporadic groups the parameter $a$ does not exceed 2 . The results of [10] show that the groups $L=M_{11}(k(L)=10), L=M_{22}(k(L)=12), L=M_{23}$ $(k(L)=17)$, and $L=M_{24}(k(L)=26)$ do not satisfy inequality (6). For the remaining sporadic simple groups, except the Mathieu groups, the calculation is obvious.

\subsection{Exceptional simple groups of Lie type.}

Lemma 13. Let $L$ be an exceptional simple group of Lie type satisfying the inequality of Lemma 10. Then $L \simeq G_{2}(2)^{\prime}$.

Proof. We consider the Steinberg character to exclude various possibilities.

1. Let $L$ be isomorphic to the group ${ }^{2} B_{2}(q), q=2^{t}$. By [18, we have $k(L)=q+3$. By Lemma 10, we obtain

$$
q^{2} \leq \chi_{0}(1) \leq 1.733 t(q+3)
$$

It is easy to show that this inequality is not fulfilled for any $t \geq 3$.

2. Let $L$ be isomorphic to ${ }^{2} G_{2}(q), q=3^{t}, t \geq 3$. In this case, the inequality has the form

$$
q^{3} \leq \chi_{0}(1) \leq 1.733 t(q+8)
$$

(see [18, p. 500]). It is easily seen that this inequality is not fulfilled for any $t \geq 3$.

3. Let $L$ be isomorphic to ${ }^{2} F_{4}(q)^{\prime}, q=2^{t}, t \geq 1$. In this case, the inequality takes the form

$$
q^{12} \leq \chi_{0}(1) \leq 1.733 t\left(q^{2}+4 q+17\right)
$$

(see [18, p. 500]). For $q=2$, we have $k\left({ }^{2} F_{4}(2)^{\prime}\right)=22$ and $a=2$. It is easy to check that this inequality is not fulfilled for any $t \geq 1$.

4. Let $L$ be isomorphic to ${ }^{3} D_{4}(q), q=p^{t}, t \geq 1$. We use Lemma 3. In this case, the inequality has the form

$$
q^{12} \leq \chi_{0}(1) \leq 2 \cdot 3 t(6 q)^{4} \leq 6 t q^{8}
$$

with $q \geq 7$. Therefore, $p^{4 t} \leq 6 t$, which, obviously, is not true. Thus, $q \leq 5$. For $q=5$, we have $(6 q)^{4} \leq q^{9}$ and $t=1$. Hence, $5^{3} \leq 6$, a contradiction. Let $q=4$ and $t=2$. In this case we obtain $2^{24} \leq 12(24)^{4}$, i.e., $2^{10} \leq 3^{5}$, a contradiction. Thus, $q=2$ or $q=3$ and $t=1$. Consequently, the possible candidates are the groups ${ }^{3} D_{4}(2)$ and ${ }^{3} D_{4}(3)$. However, $k\left({ }^{3} D_{4}(2)\right)=35$, which rules out this group by inequality (4). By [21], we have $k\left({ }^{3} D_{4}(3)\right)=37$. Using Lemma 10 , we obtain a contradiction also in this case.

5 . Let $L$ be isomorphic to $G_{2}(q), q=p^{t}, t \geq 1$. By Lemma 1 , the inequality takes the form

$$
q^{6} \leq \chi_{0}(1) \leq 1.733 t(3, p)(6 q)^{2},
$$

which is equivalent to

$$
p^{4 t} \leq 1.733 \cdot 36 \cdot(3, p) t<64 \cdot(3, p) \cdot t .
$$

If $p \neq 3$, then $p^{4 t}<64 t$ is valid only for $p=2$ and $t=1$, i.e., $L=G_{2}(2)^{\prime} \simeq U_{3}(3)$, as stated above.

Thus, $p=3$. It is easily seen that $t=1$ in this case, whence $L \simeq G_{2}(3)$. By [10, we have $k(L)=23$. Obviously, in this case the inequality is not fulfilled. 
6. Let $L$ be isomorphic to $F_{4}(q), E_{6}(q),{ }^{2} E_{6}(q), E_{7}(q)$, or $E_{8}(q)$ with $q=p^{t}$ and $t \geq 1$. By using Lemmas 1 and 10, these cases can easily be ruled out. The lemma is proved.

\subsection{Classical simple groups of Lie type.}

Lemma 14. Let $L$ be a classical simple group of Lie type for which the inequality of Lemma 10 is valid. Then $L \simeq L_{3}^{\varepsilon}(q)$, where $3 \leq q \leq 8$ and $q \neq 7$, with the exception of $L_{3}(5)$, or $L \simeq L_{2}(q)$.

Proof. We prove the lemma by considering each series of classical groups separately and, as a rule, applying the Steinberg character as a test. Below, $q=p^{t}$, where $p$ is a prime.

1 . The case of the groups $L_{n}^{\varepsilon}(q), n \geq 3$. First, we prove that $n \leq 6$. For this, we use the estimate of Lemma 3. Inequality (4) has the following form:

$$
q^{n(n-1) / 2} \leq 2 \cdot 2(n, q-\varepsilon \cdot 1) t(6 q)^{n-1},
$$

where $q=p^{t}$. It is easily seen that $2 t \leq q$. For $q \geq 3$, we have $6 q<q^{3}$ and $q^{n(n-1) / 2}<$ $q^{3 n}$, whence $n \leq 6$. Now, assume that $q=2$. Then, using similar considerations, we can easily show that $n \leq 7$. Table 5 in [19] gives the value of $k\left(U_{7}(2)\right)$, and Table 4 gives the value of $k\left(L_{7}(2)\right)$, which show that inequality (4) fails in this case.

Let $n \leq 6$. By Lemma 3, we have $k(L) \leq 2 \cdot q^{n-1}$ for $q \geq 3$. Therefore, inequality (4) takes the form

$$
q^{n(n-1) / 2} \leq 4(n, q-\varepsilon \cdot 1) \cdot 2 q^{n-1} t .
$$

For $n \geq 7$, we have $8 t(n-\varepsilon \cdot 1)<q^{2}$. Therefore, inequality (4) implies that

$$
n^{2}-n<2 n+2 .
$$

Hence, $n \leq 3$. Using Lemma 3 (and exact values for the number of conjugacy classes), we prove Lemma 14 in the case under consideration. If $q=2$, we again use Lemma 3 and $[19$.

Considering the exact values for the remaining groups, we obtain the following list of groups isomorphic to $L_{3}^{\varepsilon}(q)$ and satisfying the inequality of Lemma 10: $L_{3}(2), L_{3}(3)$, $L_{3}(4), L_{3}(8), U_{3}(3), U_{3}(4), U_{3}(5)$, and $U_{3}(8)$.

Among the remaining groups in question, only one group $U_{4}(2)$ satisfies the inequality of Lemma 10 with the Steinberg character in the role of $\chi_{0}$. However, there is a character of degree 81 for which the inequality fails. Therefore, the final list of groups satisfying the inequality is exhausted by the above-mentioned groups $L_{3}(q)$ and $U_{3}(q)$ for $3 \leq q \leq 8$, with the exception of $q=7$ and $L_{3}(5)$. The group $L_{3}(2)$ is ruled out because it is isomorphic to $L_{2}(7)$, and the group $U_{3}(2)$ is solvable.

2. The case of the groups $B_{n}(q)$ and $C_{n}(q)$. We consider the groups $L=P \Omega_{2 n+1}(q)$ and $L=P S p_{2 n}(q)$. In the Lie notation, these are $B_{n}(q)$ and $C_{n}(q)$. By Lemma 1 , $k(L) \leq(6 q)^{n}$ in both cases, and the inequality of Lemma 10 has the form

$$
q^{n^{2}} \leq 1.733 \cdot 2 \cdot t(6 q)^{n} .
$$

If $n \geq 4$, then there are no solutions. Let $n=3$. Then $q^{9} \leq 3.466 \cdot 6^{3} \cdot q^{3}$, i.e., $q^{6} \leq 3.466 \cdot t \cdot 6^{3}$. It is easily seen that $q \leq 3$, i.e., the possible exceptions are $P \operatorname{Sp}_{6}(q)$ or $P \Omega_{7}(q)$ for $q \in\{2,3\}$.

We prove that the groups $B_{3}(q)$ and $C_{3}(q)$ for $q \in\{2,3\}$ do not satisfy the inequality in question. The results of 10 show that $k(G) \leq 98$ for each of the above-mentioned groups over $G F(3)$, and the inequality $3^{9} \leq 3.466 \cdot 98 \leq 346$ obviously fails. If $L \simeq B_{3}(2)$ or $C_{3}(2)$, then $k(L)=30$. Here, $|\operatorname{Out}(L)|=1$ for both groups, and the inequality takes the form $2^{9} \leq 1.733 \cdot 30$, which is obviously false. 
Let $n=2$. Then $q^{2} \leq 3.466 \cdot t \cdot 6^{2}$. We recall that $q=p^{t}$. Let $t=1$. Then $q=p \leq 11$. For $t=2$, the solutions are $q \in\{4,9\}$. Finally, if $t \geq 3$, then only $q=2^{t}$ with $t \in\{3,4\}$ can be a solution of the above inequality.

Now, we rule out the remaining simple groups for $n=2$. We know that the groups $C_{2}(q)$ and $B_{2}(q)$ coincide. We apply the computer algebra system GAP with the function NrConjugacyClasses (G), where we use the function ProjectiveSymplecticGroup $(4, q)$ instead of G. Now the inequality can be rewritten as

$$
q^{4} \leq 1.733 \cdot\left|\operatorname{Out}\left(C_{2}(q)\right)\right| k\left(C_{2}(q)\right) .
$$

The resulting values for the classes are summarized in the table below.

\begin{tabular}{|c||c|c|c|c|c|c|c|c|c|}
\hline$q$ & 2 & 3 & 4 & 5 & 7 & 8 & 9 & 11 & 16 \\
\hline$k\left(C_{2}(q)\right)$ & 11 & 20 & 27 & 34 & 52 & 83 & 74 & 100 & 291 \\
\hline$\left|\operatorname{Out}\left(C_{2}(q)\right)\right|$ & 2 & 2 & 4 & 2 & 2 & 6 & 4 & 2 & 8 \\
\hline
\end{tabular}

In all cases except for $C_{2}(2)$, the inequality fails. We have $C_{2}(2) \simeq S_{6}$ and $C_{2}(2)^{\prime} \simeq L_{2}(9)$. Thus, the group $C_{2}(2)$ satisfies the conclusion of the lemma.

3. The case of the groups $D_{n}(q)$ and ${ }^{2} D_{n}(q)$. In both cases, we have $n \geq 4$, because the groups of smaller Lie rank are isomorphic to the groups considered above. Since the automorphism group of $D_{4}(q) \simeq P \Omega_{8}^{+}(q)$ is calculated in an absolutely different way, the case where $n=4$ will be studied separately. For both groups, the inequality in question can be somewhat sharpened with the help of Lemma 1; this yields

$$
q^{n(n-1)} \leq 1.733 \cdot 4 \cdot 2 t \cdot(6 q)^{n} \leq 14 \cdot t \cdot(6 q)^{n},
$$

where $n \geq 5$ and $q=p^{t}$. It is easily seen that $(6 q)^{n} \leq q^{4 n}$. Therefore, if $n \geq 6$, then the inequality admits no solutions that are natural powers of $p$. Let $n=5$. Since $3<2^{8 / 5}$, we have $q^{20} \leq 14 \cdot t(6 q)^{5} \leq 14 \cdot t q^{18}$ if $n=5$. Hence, $q^{2}=p^{2 t} \leq 14 t$. The following values of $q$ are possible: 2,3 , and 4 . Returning to the initial inequality, we can easily check that $q=2$ and $q=3$ do not fit. In the case of $q=2$, the order of the outer automorphism group of $L$ is $2\left(4, q^{5} \pm 1\right)=2$, and so the inequality is not fulfilled.

Let $n=4$ and $L \simeq D_{4}(q)$. Since $|\operatorname{Out}(L)|=6\left(q^{4}-1,4\right) t$, the inequality implies the relation $p^{8 t} \leq q^{8} \leq \chi_{0}(1) \leq 5392.232 t$, which is impossible for $q \geq 3$. Therefore, $q=2$. By [10, we have $k\left(D_{4}(2)\right)=53$. The group $L$ has an irreducible character $\chi_{0}$ of degree 6075. Thus, this possibility is also ruled out.

If $L \simeq{ }^{2} D_{4}(q)$, then $|\operatorname{Out}(L)|=2\left(q^{4}+1,4\right) t$. Thus, we have the following inequality: $q^{12} \leq 1.733 \cdot 2\left(q^{4}+1,4\right) t \cdot(6 q)^{4}$. Hence, $q^{8} \leq 14 \cdot 6^{4} t=18144 t$. If $p \geq 5$, then this inequality has no solutions. It is easily seen that the only possible solutions are $q=2$ and $q=3$. By [10], we have $k\left({ }^{2} D_{4}(2)\right)=39$. Therefore, $2^{12} \leq 1.733 \cdot 39<80$, a contradiction. If $q=3$, then $3^{12} \leq 1.733 \cdot 4 \cdot k\left({ }^{2} D_{4}(3)\right)=1.733 \cdot 4 \cdot 190<1600$, a contradiction.

Thus, there are no simple orthogonal groups satisfying the conditions of Lemma 14 . The lemma is proved.

\section{$\S 5$. Characters and normal subgroups}

We consider the following situation. The group $G \leq \mathfrak{G}=A$ ? $S$ is a smallest order counterexample to the conclusion of Theorem 2, where $A=\operatorname{Aut}(L)$ and $L$ is a nonAbelian simple group. The subgroup $N=R_{1} \times R_{2} \times \cdots \times R_{m}$ is a minimal normal subgroup of $G$ that is a direct product of conjugate non-Abelian simple groups isomorphic to $L$, and $S$ is a solvable subgroup of $S_{m}$.

By the results of the preceding section, we may assume that $L$ is one of the following groups: $L_{3}^{\varepsilon}(3), L_{3}^{\varepsilon}(4), L_{3}^{\varepsilon}(8), U_{3}(5)$, or $L_{2}(q)$. Moreover, there exists a normal subgroup $\mathfrak{M}$ of $\mathfrak{G}$ containing $N$ such that $\mathfrak{M}=A_{1} \times A_{2} \times \cdots \times A_{m}$ with $A_{i}=\operatorname{Aut}\left(R_{i}\right)$ and 
$S=\mathfrak{G} / \mathfrak{M} \simeq G / M$. Here, $G$ acts transitively on the set $\left\{R_{1}, R_{2}, \ldots, R_{m}\right\}$, and the kernel of this action is the subgroup $M=G \cap \mathfrak{M}$.

In the case where $L \simeq L_{2}(q)$ for $q$ odd, we need yet another subgroup $M_{0}$ of $G$, which is defined as follows. We denote by $\mathfrak{M}_{0}$ the subgroup of $\mathfrak{G}$ lying in $\mathfrak{M}$ and isomorphic to the group $D_{1} \times D_{2} \times \cdots \times D_{m}$, where $D_{i} \simeq P G L_{2}(q)$, and put $M_{0}=\mathfrak{M}_{0} \cap G$.

We are interested in irreducible characters of $G$, the restriction of which to $N$ contains a certain special character of $N$. By the Clifford theory (see Chapter 6 in 12$]$ ), if $\Theta \in \operatorname{Irr}(N)$ and $\chi$ is an irreducible character of $G$, then the relation $\left[\left.\chi\right|_{N}, \Theta\right]_{N} \neq 0$ implies that

$$
\chi_{N}=e \sum_{i=1}^{l} \Theta_{i},
$$

where the $\Theta_{i}$ are characters conjugate to $\Theta$. We recall that the character conjugate to $\Theta$ by an element $g \in G$ is defined as follows: $\Theta^{g}(x)=\Theta\left(g x g^{-1}\right)$ for every $x \in G$. The number $l$ of distinct characters conjugate to $\Theta$ is equal to the index of the inertia subgroup $I_{G}(\Theta)$ of $\Theta$ (consisting of all $g \in G$ such that $\Theta^{g}=\Theta$ ), and the integer $e=e(\chi)$ divides $\left|I_{G}(\Theta): N\right|$ if $G / N$ is a solvable group. The character $\Theta \in \operatorname{Irr}(N)$ is said to be invariant if $I_{G}(\Theta)=G$.

In the following lemma, we state briefly some facts about characters induced from normal subgroups. The proofs can be found in [12, Chapter 6].

Lemma 15. Let $K$ be a normal subgroup of a finite group $X$ with solvable quotient group $X / K$, and let $\psi$ be an irreducible character of $K$. Then $\psi^{X}=\sum_{i=1}^{s} e_{i} \chi_{i}$, where $\chi_{i} \in \operatorname{Irr}(X)$, each $e_{i}$ divides $\left|I_{X}(\psi) / K\right|,\left.\chi_{i}\right|_{K}=e_{i} \sum_{j=1}^{l} \psi_{j}$, where the $\psi_{j}$ are characters conjugate to $\psi=\psi_{1}, l=\left|X: I_{X}(\psi)\right|$, and $\sum_{i=1}^{s} e_{i}^{2}=\left|I_{X}(\psi): K\right|$. If $\chi \in \operatorname{Irr}(X)$ and $\left[\left.\chi\right|_{K}, \psi\right] \neq 0$, then $\chi=\chi_{i}$ for some $i \leq s$.

The following result is due to Gallagher [13, and is given below in the same form as in [16, $\left(A_{2}^{\prime}\right)$ ]. To state this result, we need the following definition.

Definition 3. For every group $T$, we define the parameter $k_{0}(T)$ as follows. For each subgroup of $T$ (including $T$ ), we consider the number of conjugacy classes of its elements; the largest among these numbers is $k_{0}(T)$.

If $T$ is Abelian, then $k_{0}(T)=k(T)=|T|$. In most applications below, this number is not much different from $k(T)$ and gives an upper bound for $k(T)$. The following property makes the parameter $k_{0}(T)$ convenient: if $Y$ is a subgroup of $X$, then $k_{0}(Y) \leq k_{0}(X)$. Furthermore, Lemma 4 shows that if $Y$ is a normal subgroup of $X$, then $k_{0}(X) \leq$ $k_{0}(Y) k_{0}(X / Y)$.

Lemma 16. Let $K$ be a normal subgroup of a finite group $X$, and let $k_{0}(X / K) \leq r$. Let $\psi$ be an irreducible character of $K$. Then the number of irreducible characters of $X$ lying over (conjugate to) $\psi$ (we mean $\chi \in \operatorname{Irr}(X)$ such that $\left[\left.\chi\right|_{K}, \psi\right] \neq 0$ ) does not exceed $k_{0}\left(I_{G}(\psi) / K\right)$. In particular, this number does not exceed $r$.

Now, we describe the characters used in the proof of Theorem 2.

Let $\eta_{1}$ be an irreducible character of the subgroup $R_{1}$ invariant under $A_{1}$. The choice of $\eta_{1}$ will be specified later, depending on the type of the group $L \simeq R_{1}$. Since the group $G$ is transitive on the set $\left\{R_{1}, R_{2}, \ldots, R_{m}\right\}$, there exist elements $x_{1}, x_{2}, \ldots, x_{m}$ of $G$ such that $R_{1}^{x_{i}}=R_{i}$. We can define a character $\eta_{i}$ on the subgroup $R_{i}$ by $\eta_{i}\left(x_{i}^{-1} g x_{i}\right)=\eta_{1}(g)$ for all $g \in R_{1}$. If $\eta_{1}$ is an irreducible character of $R_{1}$ and $x_{1}, x_{2}, \ldots, x_{m}$ are elements of $G$ such that $R_{1}^{x_{i}}=R_{i}$, then $\eta_{i}=\eta_{1}^{x_{i}^{-1}}$ is an irreducible character of $R_{i}$. The character $\Theta=\eta_{1} \eta_{2} \cdots \eta_{m}$ is defined on $N$ as follows. If $g=g_{1} g_{2} \cdots g_{m} \in N$, where $g_{i} \in R_{i}$, then 
$\Theta(g)=\eta_{1}\left(g_{1}\right) \eta_{2}\left(g_{2}\right) \cdots \eta_{m}\left(g_{m}\right)$. If $\eta_{1}$ is invariant under $A_{1}$, then $\Theta$ is invariant under the group $M$.

Let $\Theta_{1}, \Theta_{2}, \ldots, \Theta_{l}$ be distinct characters conjugate to $\Theta=\Theta_{1}$ under the action of $G$. Each of these characters can be written as $\Theta_{i}=\Theta_{1}^{a_{i}}$ for an appropriate element $a_{i} \in \mathfrak{M}=A_{1} \times A_{2} \times \cdots \times A_{m}$. Since $G \leq \mathfrak{G}=A \curlywedge S=\mathfrak{M} \rtimes S$, every element $y \in G$ can be represented in the form $y=\sigma a$ for appropriate $a \in \mathfrak{M}$ and $\sigma \in S$. Since $\sigma$ permutes the $R_{i}$ and also the $\eta_{i}$, we obtain $\Theta^{\sigma}=\Theta$, and therefore, $\Theta^{y}=\Theta^{a}$ for some $a \in \mathfrak{M}$.

Thus, the number of characters conjugate to $\Theta=\Theta_{1}$ in $G$ satisfies the inequality $l=\left|G: I_{G}(\Theta)\right| \geq\left|M: I_{M}(\Theta)\right|$.

Now, we assume that the character $\Theta$ is invariant in $G$. Applying Lemma 15, we conclude that $\Theta^{G}=\sum_{i=1}^{r} e_{i} \chi_{i}$, where the $\chi_{i}$ are irreducible characters of $G$ such that $\left.\chi_{i}\right|_{N}=e_{i} \Theta$ and $r$ is the number of irreducible characters such that the decompositions of their restrictions to $N$ contain $\Theta$. By Lemmas 2, 4, and 16, we obtain $r \leq k_{0}(M / N)$. $3^{(m-1) / 2}$, where $r_{1}=k_{0}(M / N)$ is a number such that every subgroup of $M / N$ has at most $r_{1}$ classes of conjugate elements.

Since $G$ is an $A S R$-group, we have $\chi_{i}^{2}=\sum_{k} c_{k, i} \chi_{k}$, where $c_{k, i} \in\{0,1\}$ for all $i$ and $k$.

We choose an irreducible character $\psi=\chi_{j_{0}}$ of $G$ for which the coefficient $\left[\left.\psi\right|_{N}, \Theta\right]=$ $e_{j_{0}}=e$ is maximal. Then $\psi^{2}=\sum_{k} u_{k} \chi_{k}$, where the coefficients $u_{k}=c_{k, j_{0}}$ belong to the set $\{0,1\}$ and the summation is taken over all irreducible characters of $G$. It is obvious that $\psi^{2}$ is a class function. Thus, by the Frobenius reciprocity law, we obtain

$$
\left[\left.\psi^{2}\right|_{N}, \Theta\right]_{N}=\left[\psi^{2}, \Theta^{G}\right]=\left[\sum_{k} u_{k} \chi_{k}, \sum_{j=1}^{r} e_{j} \chi_{j}\right]=\sum_{j=1}^{r} u_{j} e_{j} .
$$

It is easily seen that, for every $i \leq m$, the character $\eta_{i}^{2}$ on the subgroup $R_{i}$ has the expansion $\eta_{i}^{2}=d \eta_{i}+\varpi_{i}$, where $d$ is an integer depending only on the type of the group and on the choice of $\eta_{1}$, and $\varpi_{i}$ is the sum of the remaining characters of $R_{i}$ entering this expansion with nonnegative integral coefficients (or is identically zero). Since $\left.\psi^{2}\right|_{N}=\left.\left.\psi\right|_{N} \psi\right|_{N}=e^{2} \Theta^{2}=e^{2} \eta_{1}^{2} \eta_{2}^{2} \cdots \eta_{m}^{2}$ and $\eta_{i}^{2}=d \eta_{i}+\varpi_{i}$, we can write

$$
\left.\psi^{2}\right|_{N}=e^{2} d^{m} \Theta+\Upsilon
$$

where $\Upsilon$ is a character of $N$ (or the function that vanishes identically on $N$ ). In particular, we arrive at the inequality

$$
e^{2} d^{m} \leq\left[\left.\psi^{2}\right|_{N}, \Theta\right]_{N}=\sum_{j=1}^{r} u_{j} e_{j} \leq r e \leq k_{0}(M / N) \cdot 3^{m / 2} e .
$$

Finally, we obtain the estimate

$$
e \cdot d^{m} \leq k_{0}(M / N) \cdot 3^{m / 2} .
$$

Lemma 17. Let $G$ be a minimal counterexample to Theorem 2. Then a minimal normal subgroup $N$ of $G$ is the direct product of simple groups isomorphic to $L_{2}(q)$ for some $q$.

Proof. We consider each of the possible situations separately.

Let $L \simeq L_{3}(3)$. Then $|\operatorname{Out}(L)|=2$. Therefore, $k_{0}(M / N) \leq 2^{m}$. By [10], this group has an invariant character $\eta$ of degree 39 , and the multiplicity $d$ of $\eta=\chi_{12}$ (in the notation of [10]) in $\eta^{2}$ is equal to 11 . Substituting this in (9), we get a contradiction.

Now, assume that $L \simeq L_{3}(4)$. The Steinberg character $\eta=S t_{L}$ of $L$ has degree 64 . Using the character table of this group given in [10], we see that $S t_{L}^{2}=13 \cdot S t_{L}+\varsigma$, where $\varsigma$ is the sum of the remaining characters involved in the decomposition of $S t_{L}^{2}$. The group of outer automorphisms of $L$ is isomorphic to the dihedral group of order 12 . It is easy to check that the number of conjugacy classes of any subgroup of this group does not exceed 
6. Using induction based on Lemma 4, we can easily prove that $k_{0}(M / N) \leq 6^{m}$. In this case, inequality (9) has the form $e \cdot 13^{m} \leq 6^{m} 3^{m / 2}$. Hence, $13 \leq 6 \sqrt{3}$, a contradiction.

Let $L \simeq U_{3}(3)$. By [10, we have $|\operatorname{Out}(L)|=2$, and the character $\eta=S t_{L}$ has multiplicity $d=4$ in the expansion into irreducible characters of $L$. Applying formula (9), we conclude that $e 4^{m} \leq 2^{m} 3^{m / 2}$, and this rules out the case under consideration.

Let $L \simeq U_{3}(5)$. By [10], we have $|\operatorname{Out}(L)|=6$, and the character $\eta=S t_{L}$ has multiplicity $d=16$ in the expansion into irreducible characters of $L$. Applying formula (9), we conclude that $e \cdot 16^{m} \leq 6^{m} 3^{m / 2}$, a contradiction.

Let $L \simeq U_{3}(8)$. By $[10$, we have $|\operatorname{Out}(L)|=18$, and the group $\operatorname{Out}(L)$ is isomorphic to the direct product of $S_{3}$ and a cyclic group of order 3. Using induction and Lemma 4 , we see that the number $k_{0}(M / N)$ does not exceed $9^{m}$. The character $\eta=S t_{L}$ has multiplicity $d=25$ in the expansion into irreducible characters of $L$. Applying formula (9), we obtain $e \cdot 25^{m} \leq 9^{m} 3^{m / 2}$, i.e., $25 \leq 9 \sqrt{3}$, a contradiction.

In the remaining cases $L \simeq L_{3}(8)$ and $U_{3}(4)$, we use a noninvariant character $\eta$ of the corresponding group.

In more detail, let $\eta$ be an irreducible character of $L$ of the greatest degree. For $L \simeq L_{3}(8)$ this is the character $\chi_{72}$ of degree 657 , and for $U_{3}(4)$ this is the character $\chi_{22}$ of degree 75. By [10, these characters of $L$ have the property $\eta^{2}=d \eta+\varpi$, where $\varpi$ is the sum of the remaining characters involved in the expansion of $\eta^{2}$. Simple calculations, based on the character tables of the above-mentioned groups and the GAP system, show that $d=18$ for $L \simeq L_{3}(8)$ and $d=7$ for $L \simeq U_{3}(4)$.

Let $\eta_{1}$ be the character of $R_{1}$ corresponding to the character $\eta$ of the group $L$ isomorphic to $R_{1}$. As before, we consider the character $\Theta=\eta_{1} \eta_{2} \cdots \eta_{m}$ of $N$. Let $\Theta_{1}, \Theta_{2}, \ldots$, $\Theta_{l}$ be distinct characters conjugate to $\Theta=\Theta_{1}$ under the action of the subgroup $G$.

As noted above, the number of characters conjugate to $\Theta=\Theta_{1}$ in $G$ satisfies the inequality $\left|G: I_{G}(\Theta)\right| \geq\left|M: I_{M}(\Theta)\right|$. We also note that, for all $i \leq m$ and $a \in A_{i}$, the character $\eta_{i}^{a}$ is an irreducible character of $R_{i}$ and $\left(\eta_{i}^{a}\right)^{2}=d \eta_{i}^{a}+\varpi^{a}$, i.e., the multiplicity of $\eta_{i}^{a}$ in $\left(\eta_{i}^{a}\right)^{2}$ is the same as the multiplicity of $\eta_{i}$ in $\eta_{i}^{2}$. In particular, for all $b \in A$ we have $\left(\Theta^{b}\right)^{2}=d^{m} \Theta^{b}+\Gamma$, where $\Gamma$ is the sum of the remaining irreducible characters in the expansion of $\left(\Theta^{b}\right)^{2}$. By Lemma 15, we have $\Theta^{G}=\sum_{i=1}^{r} e_{i} \chi_{i}$.

Now, we find the multiplicity of the character $\chi=\chi_{i_{0}}$ for the index $i_{0}$ such that $e=e_{i_{0}}=\max _{i=1}^{r} e_{i}$. Since $G$ is an ASR-group, we see that $\chi^{2}=\sum_{k} c_{k} \psi_{j}$ is the sum of distinct irreducible characters of $G$. Therefore,

$$
\left[\chi^{2}, \Theta^{G}\right]_{G}=\sum_{j=1}^{r} c_{j} e_{j},
$$

where $c_{j} \in\{0,1\}$. Using the Frobenius reciprocity law, we obtain

$$
\left[\chi^{2}, \Theta^{G}\right]_{G}=\left[\left.\chi^{2}\right|_{N}, \Theta\right]_{N}=e^{2}\left[\left(\sum_{j=1}^{l} \Theta_{j}\right)^{2}, \Theta\right] .
$$

Since $\left(\sum_{j=1}^{l} \Theta_{j}\right)^{2}=\Theta_{1}^{2}+\Xi$, where $\Xi$ is a sum of characters with nonnegative integral coefficients, we obtain $\left[\left(\sum_{j=1}^{l} \Theta_{j}\right)^{2}, \Theta\right] \geq\left[\Theta^{2}, \Theta\right]=d^{m}$. Thus,

$$
e^{2} d^{m} \leq \sum_{j=1}^{r} c_{j} e_{j} \leq e r \leq e|M: N| k_{0}(G / M) .
$$

Applying inequality (10) to the case where $R_{1} \simeq L \simeq U_{3}(4)$, we get $e \cdot 7^{m} \leq 4^{m} 3^{m / 2}$, whence $7 \leq 1.733 \cdot 4$, a contradiction.

If $R_{1} \simeq L \simeq L_{3}(8)$, then $18^{m} \leq 6^{m} 3^{m / 2}$, which is also false. The lemma is proved. 


\section{§6. The Characters of the GRoup $P G L_{2}(q)$}

If $q \geq 4$ is even, then the group $P G L_{2}(q)$ coincides with $L=L_{2}(q)$.

The character table of $D=P G L_{2}(q)$, where $q$ is odd, is taken from 8 .

When describing the character table of $D=P G L_{2}(q)$ for $q$ odd, we assume that $q \geq 7$. The following notation is used also in the case where $q$ is even, but in the latter case the group is denoted by $L$ : the groups $D$ and $L$ have cyclic subgroups generated by an element $a$ of order $q-1$, by an element $b$ of order $q+1$, and by an element $u$ of order $p$, where $p$ is the characteristic of $G F(q)$. Here, every element of $D$ is conjugate to one of the elements $1, u, a^{r}(1 \leq r \leq(q-3) / 2)$ or $b^{s}(1 \leq s \leq(q-1) / 2)$, or to one of the involutions $z_{-}=a^{(q-1) / 2}$ or $z_{+}=b^{(q+1) / 2}$.

Every element of $L=L_{2}(q)=P G L_{2}(q)$ with $q$ odd is conjugate to one of the elements $1, u, a^{r}(1 \leq r \leq(q-2) / 2)$, or $b^{s}(1 \leq s \leq q / 2)$.

\begin{tabular}{|c||c|c|c|c|c|c|}
\hline & 1 & $u$ & $a^{r}$ & $z_{-}$ & $b^{s}$ & $z_{+}$ \\
\hline $1_{D}$ & 1 & 1 & 1 & 1 & 1 & 1 \\
\hline$S g n$ & 1 & 1 & $(-1)^{r}$ & $(-1)^{(q-1) / 2}$ & $(-1)^{s}$ & $(-1)^{(q+1) / 2}$ \\
\hline$S t$ & $q$ & 0 & 1 & 1 & -1 & -1 \\
\hline$S g n \cdot S t$ & $q$ & 0 & $(-1)^{r}$ & $(-1)^{(q-1) / 2}$ & $(-1)^{s+1}$ & $(-1)^{(q-1) / 2}$ \\
\hline$\varphi_{i}$ & $q+1$ & 1 & $\alpha^{i r}+\alpha^{-i r}$ & $2(-1)^{i}$ & 0 & 0 \\
\hline$\zeta_{j}$ & $q-1$ & -1 & 0 & 0 & $\beta^{j s}+\beta^{-j s}$ & $2(-1)^{j+1}$ \\
\hline
\end{tabular}

Thus, $k(D)=q+2$ and $k(L)=q+1$. We denote by $\alpha$ a primitive $(q-1)$ st root of unity and by $\beta$ a primitive $(q+1)$ st root of unity in the field of complex numbers.

Now, we proceed directly to the description of the character table of $D$. There are two linear characters (i.e., characters of degree 1), namely, $1_{D}$ (the principal character) and a character denoted by $S g n$. Besides, there is a Steinberg character $S t$ and a character $S g n \cdot S t$, both of degree $q$. The family of the characters $\varphi_{i}$ consists of $(q-3) / 2$ characters of degree $q+1$, and the family of the characters $\zeta_{j}$ consists of $(q-1) / 2$ characters each of degree $q-1$.

Now, we present the character table of the group $L=L_{2}(q)$, where $q \geq 8$ is even. The group $L$ has a unique linear character $1_{L}$ (the principal character). Besides, there is a Steinberg character $S t$ of degree $q$. The family of the characters $\varphi_{i}$ consists of $(q-2) / 2$ characters of degree $q+1$, and the family of the characters $\zeta_{j}$, each of degree $q-1$, consists of $q / 2$ characters. The character table is taken from [20]:

\begin{tabular}{|c||c|c|c|c|}
\hline & 1 & $u$ & $a^{r}$ & $b^{s}$ \\
\hline $1_{L}$ & 1 & 1 & 1 & 1 \\
\hline$S t$ & $q$ & 0 & 1 & -1 \\
\hline$\varphi_{i}$ & $q+1$ & 1 & $\alpha^{i r}+\alpha^{-i r}$ & 0 \\
\hline$\zeta_{j}$ & $q-1$ & -1 & 0 & $\beta^{j s}+\beta^{-j s}$ \\
\hline
\end{tabular}

The following lemma can be deduced from the orthogonality relations. We give an elementary proof.

Lemma 18. Let $\alpha$ be a primitive $(q-1)$ st root of unity in the field of complex numbers, and let $1 \leq j<q-1$. Let $q$ be odd. If $j$ is odd, then $S=\sum_{r=1}^{(q-3) / 2}\left(\alpha^{j r}+\alpha^{-j r}\right)=0$. If $j$ is even, then $S=-2$. If $q$ is even, then $S_{1}=\sum_{r=1}^{(q-2) / 2}\left(\alpha^{j r}+\alpha^{-j r}\right)=-1$.

Proof. Let $q$ be odd. It is easily seen that $S$ is the sum of the $j$ th powers of all $(q-1)$ st roots of unity except for $1^{j}$ and $(-1)^{j}$. Since the sum of the $j$ th powers of all $(q-1)$ st roots of unity is zero for $1 \leq j<q-1$, we obtain the claim.

The statement concerning the sum $S_{1}$ for even $q$ is proved similarly. 
Lemma 19. Let $\varphi_{i}$ be an irreducible character of $D=P G L_{2}(q)$ that has degree $q+1$, where $1 \leq i \leq(q-3) / 2$ if $q$ is odd and $1 \leq i \leq(q-2) / 2$ if $q$ is even. Then the multiplicity of the Steinberg character St in $\varphi_{i}^{2}$ is equal to 2.

Proof. Let $D=P G L_{2}(q)$, where $q$ is odd. Using the character table, we calculate $\left[\varphi_{i}^{2}, S t\right]$. We take into account the fact that the sizes of the conjugacy classes in $D$ are as follows: $\left|u^{G}\right|=q^{2}-1,\left|\left(a^{r}\right)^{G}\right|=q(q+1)(r$ takes the values from 1 to $q-3)$, and $\left|z_{-}^{G}\right|=q(q+1) / 2$. We denote by $S^{(2)}$ the sum $\sum_{r=1}^{(q-3) / 2}\left(\alpha^{j r}+\alpha^{-j r}\right)^{2}$. Then

$$
\left[\varphi_{i}^{2}, S t\right]=\frac{(q+1)^{2} q+q(q+1) S^{(2)}+2 q(q+1)}{q\left(q^{2}-1\right)} .
$$

By Lemma 17, we have

$$
S^{(2)}=\sum_{r=1}^{(q-3) / 2}\left(\alpha^{j r}+\alpha^{-j r}\right)^{2}=\sum_{r=1}^{(q-3) / 2}\left(\alpha^{j r}+\alpha^{-j r}\right)+2 \sum_{r=1}^{(q-3) / 2} 1=q-5 .
$$

Substituting this in the above relation, we obtain $\left[\varphi_{i}^{2}, S t\right]=2$.

Let $D=L=L_{2}(q)$, where $q$ is even. Then the calculation is similar, but now the sum of squares of the character values $S_{1}^{(2)}=\sum_{r=1}^{(q-2) / 2}\left(\alpha^{j r}+\alpha^{-j r}\right)^{2}$ required in the calculation of the dot product is equal to $q-3$, and there is no term corresponding to the contribution of the conjugacy class consisting of involutions. Thus, the result remains the same.

Lemma 20. (i) Let $\varphi_{l}$ be an irreducible character of $D=P G L_{2}(q)$ ( $q$ is odd) of degree $q+1(1 \leq l \leq(q-5) / 4)$, and let $D_{0}$ be a subgroup of index 2 in $D$ (isomorphic to $L_{2}(q)$ ). Then $\left.\varphi_{l}\right|_{D_{0}} \in \operatorname{Irr}\left(D_{0}\right)$.

(ii) If $\gamma$ is an automorphism of $P G L_{2}(q)$ induced by an automorphism of the field $G F(q)$ and $q \notin\{4,5,9\}$, then $\gamma$ has an exact orbit on the set of characters of $L=L_{2}(q)$ that have degree $q+1$.

Proof. (i) The description of the irreducible characters of $D_{0}=L_{2}(q)$ for odd $q$ (see [20]) shows that the restriction of $\varphi_{l}$ to $D_{0}$ can be the sum of two distinct characters of $D_{0}$ only in the case where $q \equiv 1(\bmod 4)$, and, for every $r \leq(q-3) / 2$, the value of $\varphi_{l}$ at the element $\left(a^{2}\right)^{r}$ of $L$ must be equal to $2 \cos (4 \pi l r /(q-1))=2(-1)^{r}$. It is easy to check that this is possible only if $l$ is a multiple of $(q-1) / 4$. Hence, we obtain (i).

(ii) An automorphism $\sigma$ of order $e$ of the group $P=P G L_{2}(q)$ (here we consider the cases of even $q$ and odd $q$ simultaneously) induced by an automorphism of $G F(q)$, where $q=p^{e}$, takes the character $\varphi_{j}$ to the character $\varphi_{p j}$. Every automorphism of $P$ induced by an automorphism of the field is a power of $\sigma$. Now, it suffices to take $j=1$ and to verify that all $e$ characters $\varphi_{1}, \varphi_{p}, \ldots, \varphi_{p^{e-1}}$ are distinct.

In the case where $q$ is odd, it is important for us that the automorphism $\sigma$ have an exact orbit on the set of irreducible characters of the subgroup $D_{0}$, which has index 2 in $D$. Since $D_{0}$ has index 2 in $D$, we have $a^{2} \in D_{0}$. Considering the values of $\varphi_{p^{i}}$ and $\varphi_{p^{l}}$ at $a^{2}$, we see that if $\varphi_{p^{i}}=\varphi_{p^{l}}$ for some $i<l$, then $\cos \left(4 \pi p^{i} /(q-1)\right)=\cos \left(4 \pi p^{l} /(q-1)\right)$, whence $2\left(p^{l} \pm p^{i}\right) /(q-1)=k$ for some integer $k$. Since $q-1=p^{e}-1$ and $p$ is prime to $q-1$, we conclude that $2 p^{i}\left(p^{s} \pm 1\right) /\left(p^{e}-1\right)$ is an integer for some $s \leq e-1$. Since in this case we have $2\left(p^{e-1}+1\right) \geq p^{e}$, it follows that $p=3$ and $e=2$. Consequently, if $q \neq 9$, then the automorphism $\sigma$ of order $e$ has an exact orbit on the set of characters of $D_{0}$ that have degree $q+1$. This implies the statement for an arbitrary automorphism $\gamma$. We note that if $q=5$, then the set of characters of $D_{0}$ that have degree $q+1=6$ is empty. 
If $q$ is even, then we compare the values of $\varphi_{2^{i}}$ and $\varphi_{2^{l}}$ at the element $a \in P=L$. As in the preceding case, we conclude that $\varphi_{2^{i}}$ and $\varphi_{2^{l}}$ can be equal only if $\cos \left(4 \pi 2^{i} /\left(2^{e}-1\right)\right)=$ $\cos \left(4 \pi 2^{l} /\left(2^{e}-1\right)\right)$. In other words, $2^{i+1}\left(2^{s}-1\right) /\left(2^{e}-1\right)$ is an integer for some $s \leq e-1$. Since the denominator of this fraction is odd, we see that $\left(2^{s} \pm 1\right) /\left(2^{e}-1\right)$ is an integer, which is possible only if $e=2$, i.e., if $q=4$. The lemma is proved.

\section{$\S 7$. Proof of Theorems 1 And 2}

By Lemma 9, a minimal counterexample to Theorem 1 is given by a subgroup $G$ of the wreath product $A$ \ $S=\mathfrak{G}$ such that $G$ contains the socle $N$ of $\mathfrak{G}$, where $A=\operatorname{Aut}(L)$ is the automorphism group of the non-Abelian simple group $L$ and $S$ is a solvable subgroup of the symmetry group of degree $m$.

By Lemma 17, a minimal normal subgroup $N$ of $G$ is the direct product $R_{1} \times R_{2} \times$ $\cdots \times R_{m}$ of simple groups, each one being isomorphic to the group $L=L_{2}(q)$ for some $q$, and the group $G$ acts transitively on the set $\left\{R_{1}, R_{2}, \ldots, R_{m}\right\}$. Now, we assume that $q \neq 4,5$, or 9 .

We recall that $\mathfrak{M}$ is a subgroup of $\mathfrak{G}$ normalizing each subgroup $R_{i}$. Let $\mathfrak{M}_{0}$ be a subgroup of $D_{1} \times D_{2} \times \cdots \times D_{m}$ lying in $\mathfrak{M}$, where $D_{i} \simeq P G L_{2}(q)$. Put $M=\mathfrak{M} \cap G$ and $M_{0}=\mathfrak{M}_{0} \cap G$. Next, let $x_{1}, x_{2}, \ldots, x_{m}$ be elements of $G$ such that $R_{1}^{x_{i}}=R_{i}$.

The subgroup $\mathfrak{M}_{0}$ has the character $\Theta=\eta_{1} \eta_{2} \cdots \eta_{m}$, where $\eta_{1}=\varphi_{1}$ is an irreducible character of degree $q+1$ (see the notation in the preceding section) of $D_{1} \simeq P G L_{2}(q)$, and the characters $\eta_{i}$ of $D_{i}$ are defined by the formula

$$
\eta_{i}\left(x_{i}^{-1} g x_{i}\right)=\eta_{1}(g)
$$

for all $g \in R_{1}$.

The character $\Theta$, defined at every element $y=y_{1} y_{2} \cdots y_{m} \in \mathfrak{M}_{0}$ with $y_{i} \in A_{i}$ by the equation $\Theta(y)=\eta_{1}\left(y_{1}\right) \eta_{2}\left(y_{2}\right) \cdots \eta_{m}\left(y_{m}\right)$, is an irreducible character of $\mathfrak{M}_{0}$. Since $\left.\Theta\right|_{N}$ is an irreducible character of $N=R_{1} \times R_{2} \times \cdots \times R_{m}$ by Lemma 20(i) and from what has been said above, we see that $\left.\Theta\right|_{N}$ is also an irreducible character of the intermediate subgroup $M_{0}$, which lies in $\mathfrak{M}_{0}$ and is normal in $M$.

Let $\zeta_{1}$ be the Steinberg character (of degree $q$ ) of $D_{1}$. As above, we construct an irreducible character $\Xi=\zeta_{1} \zeta_{2} \cdots \zeta_{m}$ of $\mathfrak{M}_{0}$, where the $\zeta_{i}$ are irreducible characters of degree $q$ of the group $D_{i}$. By construction, $\zeta_{i}=\zeta_{1}^{x_{i}^{-1}}$. Using Lemma 20(ii), we can easily check that $\Xi$ is a $\mathfrak{G}$-invariant character.

The special choice $\eta_{1}=\varphi_{1}$ of the character and the condition $q \notin\{4,5,9\}$ ensure that $\eta_{1}^{\gamma} \neq \eta_{1}$ for every field automorphism $\gamma$ of $P G L_{2}(q)$. It follows that, for every $a \in M \backslash M_{0}$, the character $\Theta^{a}$ is distinct from $\Theta$. As noted above, we have $\left|G: I_{G}(\Theta)\right|=$ $l \geq\left|M: M_{0}\right|=t$.

We establish yet another important property:

$$
I_{G}(\Theta) M / M \simeq I_{G}(\Theta) /\left(I_{G}(\Theta) \cap M\right)=I_{G}(\Theta) / M_{0} .
$$

Indeed, $M_{0} \leq I_{G}(\Theta) \cap M$. Since every automorphism of $M_{0}$ induced by an element of $M \backslash M_{0}$ does not fix $\Theta$, we have $I_{G}(\Theta) \cap M=M_{0}$. The fact that $I_{G}(\Theta) M / M \leq G / M$ and $I_{G}(\Theta) / M_{0}$ are isomorphic has an important consequence.

By Theorem 15, if $\chi$ is an irreducible character of $G$ such that $\left[\left.\chi\right|_{M_{0}}, \Theta\right] \neq 0$, then $\left.\chi\right|_{N}=e(\chi) \sum_{i=1}^{l} \Theta_{i}$, where $\Theta_{1}, \Theta_{2}, \ldots, \Theta_{l}$ are all characters of $M_{0}$ conjugate to $\Theta=\Theta_{1}$ and $e(\chi)$ is a nonnegative integer. We have $\Theta^{G}=\sum_{i=1}^{s} e_{i} \chi_{i}$, where $s=k_{0}\left(I_{G}(\Theta) / M\right)$ (see Lemma 16). Also, we have $\sum_{i=1}^{s} e_{i}^{2}=\left|I_{G}(\Theta): M_{0}\right| \leq|G: M|$.

Since $G / M$ is a solvable subgroup of $S \leq S_{m}$, we see that $s$ does not exceed $3^{m / 2}$ by Lemma 2. 
Let $\chi_{j}$ be an irreducible character of $G$ involved in the expansion of $\Theta^{G}$. Since $G$ is an $A S R$-group, we have $\chi_{j}^{2}=\sum_{k} \delta_{k, j} \chi_{k}$, where $\chi_{k} \in \operatorname{Irr}(G)$ and $\delta_{k, j} \in\{0,1\}$ for all $k$. On the other hand, $\Xi^{G}=\sum_{k=1}^{d} b_{k} \chi_{k}$, where $\chi_{k} \in \operatorname{Irr}(G)$ and the coefficients $b_{k}$ of the expansion play the same role as the $e_{i}$ in the expansion of $\Theta^{G}$. In particular, by the invariance of $\Xi$, we obtain

$$
\sum_{k=1}^{d} b_{k}^{2}=\left|G: M_{0}\right|=t|G / M| .
$$

Here $d$ is the total number of irreducible characters in the expansion of $\Xi^{G}$.

Hence, $\left[\chi_{j}^{2}, \Xi^{G}\right]=\sum_{i=1}^{d} \delta_{i, j} b_{i}$, where $\delta_{i, j} \in\{0,1\}$. On the other hand, by the Frobenius reciprocity theorem, we have

$$
\left[\chi_{j}^{2}, \Xi^{G}\right]_{G}=\left[\left.\chi_{j}^{2}\right|_{M_{0}}, \Xi\right]_{M_{0}}=e_{j}^{2}\left[\sum_{i=1}^{l} \sum_{k=1}^{l} \Theta_{i} \Theta_{k}, \Xi\right]_{M_{0}} .
$$

By Lemma 19, $\eta_{i}^{2}=2 \zeta_{i}+\psi_{i}$ for some character $\psi_{i}$ of $D_{i}$. Therefore, if $f \leq l$, then $\Theta_{f}^{2}=2^{m} \Xi+\Upsilon_{f}$ for some character $\Upsilon_{f}$ of $M_{0}$. Hence,

$$
\sum_{i=1}^{d} \delta_{i, j} b_{i}=\left[\chi_{j}^{2}, \Xi^{G}\right]_{G} \geq e_{j}^{2} l 2^{m} .
$$

By Lemma 16, the number $d$ of irreducible characters of $G$ whose restriction to $M_{0}$ contains $\Xi$ does not exceed a number $r\left(=k_{0}\left(G / M_{0}\right)\right)$ such that each subgroup of $G / M_{0}$ has at most $r$ conjugacy classes.

The group $G / M_{0}$ is an extension of $M / M_{0}$ by the group $S$, which is a solvable subgroup of $S_{m}$. Therefore, Lemmas 2(i) and 4 imply that $k_{0}\left(G / M_{0}\right) \leq t 3^{m / 2}$. Since each subgroup of $G / M_{0}$ is an extension of an Abelian group of order dividing $t$ by a solvable subgroup of $S_{m}$, we have $d \leq t 3^{m / 2}$.

Applying the Cauchy-Bunyakowski-Schwarz inequality to (11), we obtain

$$
e_{j}^{2} l 2^{m} \leq t^{1 / 2} 3^{m / 4}\left(\sum_{i=1}^{d} b_{i}^{2}\right)^{1 / 2}=t^{1 / 2} 3^{m / 4}\left|G / M_{0}\right|^{1 / 2} .
$$

However, $\left|G / M_{0}\right|=l\left|I_{G}(\Theta): M_{0}\right|=l \sum_{i=1}^{s} e_{i}^{2} \leq l s e^{2}$, where $e=\max _{i=1}^{s} e_{i}$. We recall that $s \leq 3^{m / 2}$.

Consequently,

$$
e_{j}^{2} l 2^{m} \leq 3^{m / 2} t^{1 / 2} l^{1 / 2} e .
$$

This inequality is valid for all $j$. Choosing $e_{j}$ equal to $e$, from (12) we deduce that

$$
e(l / t)^{1 / 2} 2^{m} \leq 3^{m / 2} .
$$

Since $e \geq 1$ and $l \geq t$, we have $2^{m} \leq 3^{m / 2}$. The contradiction obtained shows that $G$ cannot be an $A S R$-group unless $R_{1}$ is isomorphic to $L_{2}(5) \simeq L_{2}(4)$ or to $L_{2}(9)$. This completes the proof of Theorem 2.

As pointed out in the Introduction, Theorem 1 follows directly from Theorem 2.

Almost all simple groups are not $A S R$-groups (the character tables for $A_{5}, S_{5}$, and $A_{6}$ are well known, and no other composition factors can occur, by Theorem 1). The subgroups of the automorphism group of $A_{6}$ that strictly contain $A_{6}$ are the following: $S_{6}, P G L_{2}(9), M_{10}$ (the stabilizer of a point in the Mathieu group $M_{11}$ ), and the group $P \Gamma L_{2}(9)$. The number of classes of all such groups, excluding the last of them, does not exceed 11, and each group has an irreducible character of degree 10. By Corollary 2 , this implies that none of these groups is an $A S R$-group. The group $P \Gamma L_{2}(9)$ has 13 
conjugacy classes and an irreducible character of degree 20, which rules out this group as well. Thus, the order of a nonsolvable $A S R$-group (if it exists) must be divisible by 3600 .

\section{$\S 8$. Examples}

In the following examples, we used the computer algebra system GAP [14]. GAP contains the library SmallGroups, which consists of small finite groups sorted by order. The call instruction looks like this: $G:=\operatorname{Small} \operatorname{Group}(m, n)$; , where $m$ is the order of a group and $\mathrm{n}$ is its number in the set of groups of order $m$. The command IdSmallGroup (G); gives the order and the number of $G$ in the form $[\mathrm{m}, \mathrm{n}]$. The command Display (CharacterTable(G)); outputs the character table of G.

In what follows, $G_{[m, n]}$ means the group SmallGroup(m,n).

Example 1. The group $G_{[64,190]}$ has the following presentation:

$$
G_{[64,190]}=\left\langle a, b, c \mid a^{16}=b^{2}=c^{2}=[b, c]=1, a^{b}=a^{-1}, a^{c}=a^{7}\right\rangle .
$$

In other words, $G_{[64,190]}=\langle a\rangle \rtimes\langle b, c\rangle \simeq C_{16} \rtimes E_{4}$. Next, we have $k\left(G_{[64,190]}\right)=16$ and $k\left(G_{[64,190]} / Z\left(G_{[64,190]}\right)\right)=k\left(C_{2} \times D_{16}\right)=14$, where $Z\left(G_{[64,190]}\right)=\left\langle a^{8}\right\rangle$. All irreducible characters of $G_{[64,190]}$ have degrees 1,2 , or 4 . The two irreducible characters of this group that are not characters of $G_{[64,190]} / Z\left(G_{[64,190]}\right)$ have degree 4.

All characters of $G_{[64,190]}$ are real, and the squares of irreducible characters can be expanded as sums of irreducible characters with coefficients 0 and 1 . At the same time, some characters enter the expansion of the tensor product of two irreducible characters of degree 4 with the coefficient 2 . Thus, this group is a real $A S R$-group but not an $S R$-group.

Example 2. The group $G_{[72,24]}$ has the following presentation:

$$
\begin{aligned}
G_{[72,24]}=\langle a, b, c, d| a^{3} & =b^{3}=c^{4}=d^{4}=[a, b]=[a, d]=[b, c]=1, \\
a^{c} & \left.=a^{-1}, b^{d}=b^{-1}, c^{d}=c^{-1}, c^{2}=d^{2}\right\rangle .
\end{aligned}
$$

Thus, $G_{[72,24]}=\langle a, b\rangle \rtimes\langle c, d\rangle \simeq E_{9} \rtimes Q_{8}$. The group $G_{[72,24]}$ is supersolvable. It can be proved that this group is also an $A S R$-group but not an $S R$-group. It has three pairs of distinct irreducible characters such that the tensor product of characters in each pair contains characters of multiplicity 2 .

To obtain an example of a nonreal $A S R$-group, it suffices to consider the direct product of a group from the above examples by the semidihedral group $S D_{2^{n}}$, where $n \geq 4$.

Examples 1 and 2 show that the absence of multiplicities greater than 1 in the decomposition of the tensor square of an arbitrary irreducible representation does not imply that the same property is valid for the tensor product of two arbitrary irreducible representations.

\section{REFERENCES}

[1] E. Bannai and T. Ito, Algebraic combinatorics. I. Association schemes, The Benjamin/Cummings Publ. Co., Inc., Menlo Park, CA, 1984. MR0882540 (87m:05001)

[2] G. D. James, The representation theory of the symmetric groups, Lecture Notes in Math., vol. 682, Springer, Berlin, 1978. MR0513828 (80g:20019)

[3] L. S. Kazarin and V. V. Yanishevskiǔ, SR-groups of order $2^{n} p$, Mathematics in Yaroslavl' Univ., Yaroslavl', 2006, pp. 257-262. (Russian)

[4] A. I. Kostrikin, Introduction to algebra. 3. Fundamental structures of algebra, "Nauka", Moscow, 2000. (Russian)

[5] S. P. Strunkov, On the ordering of characters of simply reducible groups, Mat. Zametki 31 (1982), no. 3, 357-362; English transl., Math. Notes 31 (1982), no. 3-4, 182-184. MR0652840 (83e:20016) 
[6] M. Hamermesh, Group theory and its application to physical problems, Addison-Wesley Publ. Co., Inc., Reading, MA-London, 1962. MR0136667 (25:132)

[7] M. Hall, Combinatorial theory, 2nd ed., John Wiley and Sons, Inc., New York, 1986. MR0840216 (87j 05001)

[8] J. Bierbrauer, The uniformly 3-homogeneous subsets of $P G L(2, q)$, J. Algebraic Combin. 4 (1995), 99-102. MR1323743 (95m:20004)

[9] R. W. Carter, Finite groups of Lie type. Conjugacy classes and complex characters, John Wiley and Sons, Inc., New York, 1985. MR0794307 (87d:20060)

[10] J. H. Conway, R. T. Curtis, S. P. Norton, R. A. Parker, and R. A. Wilson, Atlas of finite groups, Oxford Univ. Press, Eynsham, 1985; http://brauer.maths.qmul.ac.uk/Atlas/v3/ MR0827219 (88g:20025)

[11] J. D. Dixon, The Fitting subgroup of a linear solvable group, J. Austral. Math. Soc. 7 (1967), 417-424. MR0230814 (37:6372)

[12] I. M. Isaacs, Character theory of finite groups, Pure Appl. Math., No. 69, Academic Press, New York-London, 1976. MR 0460423 (57:417)

[13] P. X. Gallagher, The number of conjugacy classes in a finite group, Math. Z. 118 (1970), 175-179. MR 0276318 (43:2065)

[14] The GAP Group, GAP — groups, algorithms and programming, version 4.4.9, Aachen, St. Andrews, 2006, http://www.gap-system.org

[15] L. S. Kazarin and I. A. Sagirov, On degrees of irreducible characters of finite simple groups, Proc. Steklov Inst. Math. 2001, Algebra Topology, suppl. 2, S71-S81. MR2067924 (2005f:20017)

[16] L. G. Kovács and G. R. Robinson, On the number of conjugacy classes of a finite group, J. Algebra 160 (1993), 441-460. MR 1244923 (94m:20057)

[17] M. W. Liebeck, C. E. Praeger, and J. Saxl, The maximal factorizations of the finite simple groups and their automorphism groups, Mem. Amer. Math. Soc. 86 (1990), no. 432. MR1016353 (90k:20048)

[18] M. Liebeck and L. Pyber, Upper bounds for the number of conjugacy classes of a finite group, J. Algebra 198 (1997), 538-562. MR.1489911 (99c:20023)

[19] I. G. Macdonald, Numbers of conjugacy classes in some finite classical groups, Bull. Austral. Math. Soc. 23 (1981), no. 1, 23-48. MR0615131 (82j:20092)

[20] J. McKay, The non-abelian simple groups $G,|G| \leq 10^{6}$ - character tables, Comm. Algebra 7 (1979), no. 13, 1407-1445. MR0539357(80e:20024)

[21] N. Spaltenstein, Caractères unipotents de ${ }^{3} D_{4}\left(F_{q}\right)$, Comment. Math. Helv. 57 (1982), 676-691. MR:0694610 (84k:20018)

Mathematics Department, Yaroslavl Demidov State University, Sovetskaya 14, Yaroslavl 150000, RUSSIA

E-mail address: kazarin@uniyar.ac.ru

Mathematics Department, Yaroslavl Demidov State University, Sovetskaya 14, Yaroslavl 150000, Russia

E-mail address: yvitaliy@rambler.ru

Received 14/FEB/2007

Translated by B. M. BEKKER 